\title{
The Politico-Economic Challenges of Ghana's National Health Insurance Scheme Implementation
}

\author{
Adam Fusheini $i^{1,2^{*}}$
}

\section{Abstract}

Background: National/social health insurance schemes have increasingly been seen in many low- and middle-income countries (LMICs) as a vehicle to universal health coverage (UHC) and a viable alternative funding mechanism for the health sector. Several countries, including Ghana, have thus introduced and implemented mandatory national health insurance schemes (NHIS) as part of reform efforts towards increasing access to health services. Ghana passed mandatory national health insurance (NHI) legislation (ACT 650) in 2003 and commenced nationwide implementation in 2004. Several peer review studies and other research reports have since assessed the performance of the scheme with positive rating while challenges also noted. This paper contributes to the literature on economic and political implementation challenges based on empirical evidence from the perspectives of the different category of actors and institutions involved in the process.

Methods: Qualitative in-depth interviews were held with 33 different category of participants in four selected district mutual health insurance schemes in Southern (two) and Northern (two) Ghana. This was to ascertain their views regarding the main challenges in the implementation process. The participants were selected through purposeful sampling, stakeholder mapping, and snowballing. Data was analysed using thematic grouping procedure.

Results: Participants identified political issues of over politicisation and political interference as main challenges. The main economic issues participants identified included low premiums or contributions; broad exemptions, poor gatekeeper enforcement system; and culture of curative and hospital-centric care.

Conclusion: The study establishes that political and economic factors have influenced the implementation process and the degree to which the policy has been implemented as intended. Thus, we conclude that there is a synergy between implementation and politics; and achieving UHC under the NHIS requires political stewardship. Political leadership has the responsibility to build trust and confidence in the system by providing the necessary resources and backing with minimal interference in the operations. For sustainability of the scheme, authorities need to review the exemption policy, rate of contributions, especially, from informal sector employees and recruitment criteria of scheme workers, explore additional sources of funding and re-examine training needs of employees to strengthen their competences among others.

Keywords: Politico-Economic Challenges, Health Insurance, Implementation, Ghana

Copyright: (๑) 2016 by Kerman University of Medical Sciences

Citation: Fusheini A. The politico-economic challenges of Ghana's national health insurance scheme implementation. Int J Health Policy Manag. 2016;5(9):543-552. doi:10.15171/ijhpm.2016.47

\section{Article History:}

Received: 28 July 2015 Accepted: 23 April 2016 ePublished: 27 April 2016

View Video Summary

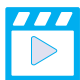

*Correspondence to:

Adam Fusheini

Email: adam.fusheini@gmail.com

\section{Key Messages}

Implications for policy makers

- Within the context of this study, addressing the political and economic challenges of the national health insurance schemes (NHIS) in Ghana requires a policy review of the exemption policy to make it more target-specific. This has the potential of ensuring those who really cannot afford get services.

- Contributions from both formal and informal sector employees also need to be marginally adjusted upward including the introduction of some percentage of taxes on alcohol and tobacco products as is done in countries such as Belgium and the United Kingdom and the proceeds channelled into the National Health Insurance Fund (NHIF) as additional sources of funding.

- It is also the view of this study that the government may consider options such as reviewing the health insurance levy upwards by at least $1.5 \%-2.0 \%$ as a way of matching revenue with increased utilisation and membership. As a way of ensuring affordability and equity, some percentage of the health sector budget support could be used to extend coverage for the exempt groups and subsidize the other groups.

- Dealing with the political challenges requires policy-makers to ensure that recruitment into the scheme reflects competence and experience rather than other considerations such as political affiliation as a way of dealing with the capacity challenges confronting the scheme.

- It is also the view of this study that policy-makers consider adopting measures including making advance payments of a percentage of submitted claims by service providers as a way of minimising the reimbursement delay challenges to forestall providers running out of stock and consumables.

Implications for the public

The study demonstrates that the economic challenges in particular have serious consequences in relation to the public being ready to enrol and renew their membership of the scheme. Inability to reimburse service providers on time mean that sometimes card-bearing members of the public are turned away. Threats of suspension of services by service providers also create anxiety and uncertainty for the public. Overall, this has an implication for the health seeking behaviour of the population.

${ }^{1}$ Centre for Health Policy/MRC Health Policy Research Group, School of Public Health, Faculty of Health Sciences, University of the Witwatersrand, Johannesburg, South Africa. ${ }^{2}$ Department of Health Policy, Planning and Management, School of Public Health, University of Health and Allied Sciences, Ho, Ghana. 


\section{Background}

National/social health insurance schemes have increasingly been seen in many low- and middle-income countries (LMICs) as a vehicle to universal health coverage (UHC) and a viable alternative funding mechanism for the health sector. $^{1-3}$ Several countries, including Ghana have thus introduced mandatory national health insurance schemes (NHIS) as part of reform efforts towards increasing access to health services; and are at different stages of implementation. While mandatory contributions to NHIS are preferable, it is a major challenge to implement in countries with large informal sectors ${ }^{4}$ such as Ghana. The move towards national health insurance (NHI) aims at enforcing social equalisation, reducing out-of-pocket (OOP) payment and increasing access to healthcare. ${ }^{1,5}$ As De Allegri et al argue, the concept of insurance is applied at the micro-level to facilitate access to care and offer financial protection against the cost of illness, by favouring community resource pooling and risk-sharing. ${ }^{6}$ While health insurance schemes seek to achieve these, researchers have also drawn attention to the challenges of implementation developing countries face. ${ }^{7}$ In the literature, three major challenges including: collection of revenue, financial risk management, and spending of resources on service providers have been identified. ${ }^{8}$ Within the context of Sub-Saharan Africa, five broad challenges: ( $i$ ) lack of clear legislative and regulatory framework; (ii) low enrolment rates; (iii) insufficient risk management measures; (iv) weak managerial capacity; and $(v)$ high overhead costs ${ }^{6}$ have been noted. These hamper the successful development of various health insurance systems, especially, communitybased schemes yielding negative effects on potential progress towards increased access to care and improved financial protection. ${ }^{6}$ Other challenges include weak institutional capacity for effective management, ineffective or unenforced regulatory mechanisms, rigid administrative procedures, and entrenched customs and practices that are difficult to change. ${ }^{7}$ Furthermore, there is increasing body of literature on the technical challenges of implementing health insurance in LMICs. ${ }^{9-12}$ In a study of national health insurance (NHI) policy implementation challenges in Nepal, for instance, financial viability has been mentioned as one major factor to achieving a more comprehensive NHI system. ${ }^{4}$

In the specific context of Ghana, while much has been achieved in relation to dealing with the implementation challenges there has been failure to recognize and deal effectively with the political ${ }^{12}$ and economic challenges in the implementation of the scheme. In this study, we seek to contribute to previous studies on political and economic challenges confronting the implementation of NHIS in LMICs. We do this by presenting an internal view from the perspectives of the key actors involved in the process based on the context-specific case of Ghana. This study could help policy-makers and insurance scheme managers design approaches to deal with the economic and political challenges. This, we hope, would improve the operations of the scheme as it provides information from their perspectives. Above all, the study will not only interest others pursuing UHC through these mechanisms but also provide useful lessons to consider context-specific factors in order to avoid some of these challenges.
Summary of the Main Elements of Ghana's National Health Insurance Scheme

Ghana passed mandatory NHI Act (Act 650 of 2003, which has since been amended by Act 852 of 2012). Nationwide implementation commenced in 2004 via decentralised district-wide mutual health insurance schemes (DMHISs). The health insurance scheme was an effort at reducing impoverishment and catastrophic health expenditure. Clearly, it was an acknowledgment that the then system of healthcare (cash and carry) could not fully identify and protect the poor, vulnerable, children, aged, and other marginalised groups in the Ghanaian society. Thus, the main goal of the NHIS is to make healthcare affordable to all by removing OOP payment at the point of service, and to achieve equity of access based on need, rather than ability to pay. ${ }^{1,12,13}$

The scheme is financed from several sources including earmarked tax $(2.5 \%$ value added tax - hereafter referred to as VAT), $2.5 \%$ of the $17.5 \%$ of formal sector employees' contribution to Social Security and National Insurance Trust (SSNIT), budgetary allocation, donations, investments, grants and annual contributions from informal sector employees and formal sector employees who do not contribute to SSNIT. Membership is open to all residents of Ghana upon subscription to the scheme (exception being Ghana armed forces and police service, and those with proof of holding a private health insurance scheme).$^{14}$ The 2013 annual report of the NHIA, however, noted the addition of three categories of membership comprising Ghana police, military and security services. This three categories constitute $0.1 \%, 0.2 \%$, and $0.003 \%,{ }^{15}$ respectively, of total membership as of December 2013. Enrolment entitles members to comprehensive benefits package covering over $95 \%$ of both inpatient and outpatient services of all common illnesses in Ghana.

Since implementation, several studies have examined the working of the NHIS. For instance, some studies have shown dramatic increases in patient utilisation of health services and coverage, especially, by the poor and the disadvantaged in Ghana following the introduction of the NHIS. ${ }^{13,16}$ Such increases in utilisation have, however, not only put pressure on the available facilities in hospitals but also pose serious challenges to managers of hospitals as increases in workload; over-stressed staff, have affected quality of care. ${ }^{1,17,18}$ A number of studies report greater accessibility and fairly high levels of satisfaction with the system. ${ }^{19-22}$ Equity in healthcare through the NHIS of Nigeria and Ghana has also been the concern of Odeyemi and Nixon's study, where they noted that in spite of Ghana's uniform benefits across all beneficiaries and improvements in equity, there is a pro-rich and pro-urban bias in membership. ${ }^{23}$ The effects of the NHIS on healthcare utilisation has also engaged the attention of researchers with evidence that individuals enrolled on the scheme are more likely to obtain prescriptions, visit clinics and seek formal healthcare when sick. ${ }^{24}$ The conclusion, therefore, is that government's objective of increasing access to the formal healthcare sector has at least been partially achieved..$^{24}$ Issues of quality have also been examined with the conclusion that there has not been a corresponding improvement in health infrastructure and equipment as well as human resource to match demand for healthcare under the scheme. ${ }^{17,18}$ 
Attention has also been drawn to limitations and challengespredominantly financial sustainability, managerial problems and inadequate technology. ${ }^{1,25-28}$ Among these challenges, evidence suggests that delays in reimbursement of health facilities resulting in shortages of basic consumables continue to be a huge problem to the effective operations of health facilities as well as limited space within the hospitals to cope with the increasing number of service demands. ${ }^{1}$ Agyepong and Adjei $^{12}$ and Imurana et al, $^{29}$ identified the major implementation problems of the NHIS to include poor leadership, corruption, lack of consensus, rapidity and politicisation of implementation, lack of participation, poor sense of direction, limited understanding and management of the political challenges, weakened checks and balances and use of short cut. ${ }^{12,29}$ The political challenges have also been referred to by Abiiro and McIntyre in their analysis of the one-time premium payment proposal under the NHIS as being very controversial and highly politicised within Ghana and internationally.,31 Furthermore, the authors argue that so much confusion surrounds stakeholders' understanding of the policy issue. Again, the uncertainties surrounding the policy economically means that powerful stakeholders are yet to take clear positions on it. ${ }^{30}$

This underscores the need to explore the political and economic challenges affecting the implementation of the NHIS.

\section{Methods}

Design

The study is an embedded case study design intended to provide in-depth understanding of the main political and economic challenges confronting the implementation of the NHI scheme as identified by interview subjects. This is through an exploratory qualitative inquiry. As our aim was to explore and analyse the implementation process of the NHIS so as to produce findings derived from real world settings, where the phenomena of interest unfold naturally, ${ }^{32}$ qualitative case study approach was the most appropriate. Implementation was clearly not a simple linear progression through a series of pre-determined steps that could be studied from the lens of quantitative approaches. The study covered the implementation process from 2004-2013.

\section{Study Areas}

The study was conducted in selected districts in Northern and Southern Ghana. The districts were the Nanumba North and South in the Northern region and the Ashiedu Keteke and Osu Klottey sub-metros in the Greater Accra Region to reflect our aim of achieving a relatively fair representation of the north-south divide. The Nanumba North district has a total population of 141584 of which 101584 is rural while 40000 are urban dwellers. ${ }^{33}$ The Nanumba south district (NSD) has a total population of 93464 of which 16712 is urban while 76752 are rural. ${ }^{33}$ Ashiedu-Keteke sub-metropolitan district assembly is the smallest among the 11 sub-metropolitan districts under the Accra Metropolitan Assembly (AMA) with a population of 117525 people, which is also the figure for urban population. ${ }^{33}$ Osu Klottey sub-metro is one of $11 \mathrm{sub}$ metros within the AMA. It has a total population of $121723,{ }^{33}$ and entirely urban.

Our choice of two rural districts in the north as against two urban in the south was aimed at ascertaining the extent of implementation challenges in resource-constrained areas of the north as opposed to resource-endowed areas of the South. Thus, within the context of this study, the areas were chosen because of the acknowledgement that there are long standing inequities in healthcare access in the country, with the south benefitting more than the north. ${ }^{34-37}$ Again, it is reported that on almost all socio-economic indices, rural Ghana compares unfavourably with urban Ghana with the north-south divide the main delineation in terms of poverty. ${ }^{37,38}$ The Nanumba North and South are two rural districts in the Northern region with few medical facilities. On the other hand, the Osu Klottey and Ashiedu Keteke sub-metropolitans are located within Accra, the national capital. Both are urban and have better public and private health facilities as well as a large number of medical personnel. While earlier studies ${ }^{39}$ pointed to the considerable inequalities of health status and outcomes between urban and rural areas and the different regions of Ghana, recent studies still underlined the broad disparities between the North and the South. There is also the observation that spatial inequalities in health and socioeconomic development between Southern and Northern Ghana and between rural and urban areas ${ }^{34}$ are still a major problem and wider in the regions of Northern Ghana compared to Southern Ghana. ${ }^{40}$

\section{Sampling and Data Collection}

Thirty-three participants were involved in the in-depth interviews. Participants were purposefully selected following a review of policy documents and other secondary sources such as peer reviewed papers, theses, other published and unpublished materials, the internet and other grey literature as well as through a stakeholder mapping and snowballing. Participants included implementing agencies and service providers (NHIA; regional offices and DMHISs, service providers); politicians; interest groups/professional associations; donor partners and non-governmental organizations (NGOs); and bureaucrats. In the Northern region, a total of ten interviews were conducted comprising managers and former managers of mutual health insurance schemes, government and private health service providers, regional and former regional managers of the NHIS, and a professional association. In the Greater Accra Region of Southern Ghana, a total of seven interviews took place with scheme managers, regional and former regional managers and government health service providers. The remaining sixteen interviewees were national level actors and stakeholders and other institutional representations. All the interviews but two took place at secured locations - the work places or offices of the participants, mostly during working hours. The interviews were based on an interview guide and a protocol of questions and were specifically adapted for each category of participants and centred on the interviewees' perception and experience of the implementation process - including how the implementation was organised, actors involved and the challenges encountered. Interviews followed a 'grandtour' approach where respondents were steered towards a 
small number of key interview destination issues but also encouraged to talk in terms which came naturally to them. ${ }^{41}$ Additional notes were taken when necessary in order to give "richer meaning to the words that were spoken." ${ }^{2}$ Besides one interviewee that refused to be recorded, a number of interviewees requested some parts of the interviews to be done off-record. Each interview lasted between 30-60 minutes with the exception of three that went beyond an hour. The first author conducted all the interviews in English. Data was collected between June and December 2011 and again in February 2012. All interviews were digitally audio recorded, transcribed and analysed. Users of health services were not included in the interviews as we were more interested in the institutional representations and also considered users' perspectives as another set of research. Table shows the category of interviewees.

\section{Data Analysis}

Transcription was done with the assistance of software called Express Scribe. Transcripts were edited for grammatical errors without changes to the content. To assist analysis, a coding scheme was developed which involved placing extracts from the data in categories labelled by theme. Themes were derived from the literature review, observations made of recurring interview content and included policy design and content, political commitment to health funding reform, organization of the implementation, challenges to implementation, and the perceived impact of policy; themes were subsequently split into subthemes, association or relationships between the subthemes and a thematic map constructed which allowed for analysis of patterns, including evidence of consensus and disagreement between different stakeholders. The thematic analysis and pattern-building generated from the interview data revealed interrelated challenges - mainly political and economic confront the implementation of the NHIS in Ghana.

\section{Results}

\section{Political Challenges}

Respondents identified political challenges as an important factor to the successful implementation of the NHIS in Ghana. Political variables identified included: $(i)$ over politicisation and political interference; and (ii) time and timing of policy changes.

Table. Categories of Respondents

\begin{tabular}{lc}
\hline Category & No. of Interviewees \\
\hline Politicians & 3 \\
NHIA & 5 \\
Regional managers, NHIS & 2 \\
DMHISs & 6 \\
Service providers & 5 \\
NGOs; international actors/development partners & 5 \\
Bureaucrats & 3 \\
Interests groups/professional associations & 2 \\
Research institutes/think tanks & 2 \\
Total & 33 \\
\hline
\end{tabular}

Abbreviations: NHIA, National Health Insurance Authority; DMHIS, district mutual health insurance scheme; NGO, non-governmental organization. NHIS, national health insurance schemes.

Source: Field notes.

\section{Over Politicisation and Interference}

Respondents noted how initial implementation in particular met many challenges and bottlenecks because of scepticism and doubts as a result of politics.

"There was a lot of politics in the minds of the people accepting the health insurance policy because one group felt the programme was not theirs..." (Former regional manager, September 26, 2011).

"It was also another challenge that the whole thing was being politicised because the party in power just loads the whole place there with its people" (Trade unionist, September 28, 2011).

Supporting the view, a regional manager in Southern Ghana blamed the over politicisation on the fact that the policy emerged in the heat of politics.

"It started in the heat of a political season. So a lot of political connotation was put onto it and from the administrative point and then implementation point... a lot of political twist is put into it; and that led to the scheme not being attractive to a lot of people in the country" (Regional Manager, September 26, 2011).

Participants also claimed recruitment of health insurance staff was political and not primarily based on criteria of competence, experience, skills and qualifications as detailed in the guidelines for design and implementation of mutual health insurance schemes in Ghana.

"The political things are there because if there is a change of government there is a change of CEO and the CEO is changed according to the ruling party and not that you are putting up a technical person" (Bureaucrat, August 4, 2011). "The appointments that are coming into the positions from national up to regional and district levels are the positions that are owed power... and that for me is a worry because if there is a change of government, that means there would be changes...it doesn't give stability to running the system" (Bureaucrat, August 4, 2011).

On the strength of the above and others, majority of respondents alleged that the scheme has become a vehicle for rewarding party members. Respondents expressed the view that political clientelism has characterised the NHIS operations in terms of recruitment.

\section{Time and Timing of Policy Changes}

Respondents also alluded to the rapidity with regards to policy decisions as a major impediment to implementation. Though unsurprising as the Ghana scheme is a "moving target" and implementation, a continuous process with feedback to inform policy direction, this is rather worrisome for some stakeholders, especially, service providers who argued that such rapidity of policy changes creates anxiety and uncertainty.

"Then these other issues of policies I talked about; sometimes they set out to look at one thing then along the line, they realise that no they did not analyse it very well and so they change it along the way; so it has not been the best" (CHAG, August 16, 2011).

"And also, those there with their policies, today they are saying this and they are changing, now they want to go to capitation, I don't know which is which; whether they are yet 
to go or not. They should let us know exactly what they want us to do" (District Service Provider, December 16, 2011).

The observations highlight the uncertainty and anxiety surrounding the NHIS, which adversely affect implementation. Also, this finding highlights the fact that policy-making and implementation are embedded as opposed to the differentiation that one is political and the other administrative..$^{43-45}$

\section{Economic Challenges}

In-depth interviews also revealed lack of adequate financial resources to sustain the scheme. Interviewees were unanimous that the economic conditions of the scheme threaten its financial sustainability. Factors identified by participants as affecting the economic sustainability and maintaining the confidence of subscribers in the system include low premiums or contributions; broad exemptions; poor gatekeeper enforcement system; culture of curative and hospital-centric care; and fraud and corruption.

\section{Rates of Insurance Premiums/Contributions}

Participants across the range of categories were unanimous on the impact of the rate of contributions, especially, by informal sector employees on the sustainability of the scheme. They claimed contributions were rather low as against the cost of treatment but even in advanced countries; healthcare services are subsidized by governments.

"The sustainability of the health insurance is also a problem because the premiums are so low. You know, as at now we are told that there is only three months, or four months cover (security deposit) instead of like eight to ten months, they only have money for four months. If the government does not come in, then we don't know what will happen to the health insurance" (Development partner, December 1, 2011).

"In effect, the premiums and all the other issues did not come out of rules; but they (NPP) were more concerned about the fact that they did not want anybody to pay anything there at the healthcare delivery point so that their political argument of cash and carry that we (NDC) had could be distinguished; but of course, it also immediately showed that the thing was not sustainable" (Political Actor, August 17, 2011).

They argued that contributions are not based on actuarial calculations or on the cost of treatment but rather based on political expediency. An interviewee asserted:

"I remember one of the meetings; I got mad with one of the people because we in the premium determination committee; while we were then looking at figures and trying to play with actuarial figures, one of the members came and said oh, the government says everybody should pay between GHS 7.20p and 48 GHS. And when I asked what the basis for saying that is? He said that oh you see, the Nkoranza one is paying $3.60 p$ and that one is only for OPD. So if you are adding admissions, you just multiply it by two" (Bureaucrat, August 4, 2011).

Most participants, however, attributed the challenge to income data unreliability in Ghana, which makes it difficult to definitively assess and rate for premium contributions. This is more so as majority works in the informal sector. Practically, contributions are flat across schemes and socioeconomic groups although Act 650 of 2003 and 852 of 2012 required and still requires contribution according to ability and healthcare according to need.

Participants also noted the disconnection between increases in membership, utilisation and increases in revenue and its impact on the financial viability of the scheme.

"If you look at the level at which the schemes grow, it tells you that unless something serious is done, the current level of financing may run out. So some more financing to sustain it needs to be added" (District Scheme Manager, July 20, 2011).

"The most critical challenge that I will tell you is the sustainability of the NHI because it is becoming very clear that if we continue the way we are now by next year (2013), the Authority will not have enough funds to meet its needs. That's the sustainability component in this thing because premiums of the informal sector are really, low" (Development Partner, August 26, 2011).

What is clear from the views expressed above are that the low contributions create anxiety about the financial stability of the scheme and the uncertainty surrounding the long term financial sustainability of the programme. It also highlights the need to explore additional funding sources.

\section{Broad Exemptions}

Majority of the interview participants viewed the exemption policy of the NHIS as being too generous. Only about $30 \%$ contribute with the broad exemption.

"When you take the membership, you will see that close to 60 or $70 \%$ do not pay any contribution and so sustainability is in doubt" (a consultant, November 20, 2011).

"Creating blanket access to so many people, we should think about sustainability; really to ensure that the resources go to people who really need them. So, I do not think it is bad but the sustainability has become problematic" (Development Partner, August 26, 2011).

"It is one of the best in terms of coverage. That is the benefits package,....It covers virtually about 90\% (sic) (over 95\%) of all the diseases that affect us in the country; It has built in a lot of social mechanisms: children under 18, aged over 70, SSNIT pensioners, indigents, pregnant women. So virtually, it covers almost $60 \%$ of the population... but we are at the state of difficulty because cost containment is now becoming the biggest issue that we have because of the way that it is loaded" (Political Actor, February 14, 2012).

Much as actors recognised the need to scale up coverage so as to achieve universal access in line with policy-makers desires and international recommendations, its impact on the financial viability needs consideration.

The blanket exemption raises a number of issues: the policy is not target-specific enough as to ensure those who really need services get it. It calls into question issues of equity, which is a real challenge between the North and the South and rural and urban Ghana. This was highlighted by a respondent when he asserted that the wife and children of the Director-General of the Ghana health service, for instance, should not have the same exemption under the policy as the wife and children of a poor farmer in a rural community like Gbungbaliga ${ }^{[1]}$ just because his wife is a pregnant woman and his children are under eighteen years. 
"His children should not have the same exempt rate with somebody who is coming from Gbungbaliga, a village like that whose parents are always on the farm; people who can genuinely pay, we shouldn't exempt them. That is the policy we have to look at in order to make the scheme sustainable" (Regional Manager, July 22, 2011).

\section{Poor Gatekeeper System}

It was a prevailing view among participants in the two regions that ineffective enforcement of the gatekeeper system is undermining the financial sustainability of the scheme. In principle, clients need to enter the system at the basic primary healthcare level for initial examination and treatment of cases such as simple malaria, and should then be referred upwards to the appropriate level if necessary. In practice, however, clients bypass the lower levels, which put strain on the health insurance fund as tariffs are higher at that level.

"The gatekeeper system is not being followed. You start from the primary healthcare to the tertiary but where you have a tertiary facility within a metropolis, the clients rather prefer going to the tertiary and as soon as they go to the tertiary, we pay higher bills" (Regional Manager, July 22, 2011).

There were also concerns regarding moral hazards with nonrestrictions regarding usage. Clients now engage in frivolous use of healthcare because there is no payment at the point of service as well as non-restriction on how many times a subscriber can visit a facility.

"Our arrangement challenge also is that we did not restrict use....So again, you are getting things like what we called health shopping" (Bureaucrat, August 4, 2011).

"For example, the number of times the person should come to hospital in a year. We need to put maybe a check on it because now a whole couple of times, people come every month. A rash here they will come; a child is coughing, the next morning they will come" (Regional Medical Director, September 15, 2011).

Medical professionals have a responsibility of enforcing the gatekeeper system. However, it could be argued that three factors affect their efforts to carry out this responsibility: the lack of the requisite technology to ascertain if a client has visited primary healthcare (PHC) facility before; availability and fair geographical distribution of facilities; and medics lacking the incentive to check.

\section{Culture of Curative and Hospital-Centric Care}

Another economic challenge of the NHIS is the culture of curative and hospital-centric care that has characterised the Ghanaian health system over the years.

"In Ghana, people focus more on the curative but if you can prevent the onset of the disease itself, there would be no need to go to the hospital and pay" (Former CEO, NHIA, February 28, 2012).

"So, if you do not put in efforts by putting some aspect of the money for preventive things, then insurance would not be sustainable. Now, we are only treating people when they are sick but it is better you also prevent so that they would not even access healthcare so that you go and pay for them" (Regional Medical Director, September 15, 2011).
Fraud, Abuse and Sustainability

Fraud and abuse emerged as key characteristics of early stage implementation. Interviewees provided examples of the many dimensions: $(i)$ service providers submitting claims and receiving reimbursement for 'caesarean operations on men.' After conducting clinical audits, "what we saw was that men were being reimbursed on caesarean. Can you imagine claims bearing men being pregnant?" (Regional Manager, July 22, 2011). (ii) Rural health facilities with no capacity and accreditation for caesarean submitting claims for caesarean sections:

"You see that where in a rural setting, a community clinic where almost three-quarters of the claims, the ante-natal birth delivery are all caesarean; and you know that is not possible. In a rural clinic, they don't have that capacity. There is no caesarean surgeon to do that" (Regional Manager, July $22,2011)$.

At other times, monies were found to have been paid to non-existing providers. Clinical audits in six schemes in the Northern region exposed so much malfeasance.

"...we realized that an amount of almost 11 billion old Ghana Cedis (equivalent of US\$53538.89/£33 146201.31) was recouped back from the providers to the scheme. This shows that these were going to be bad monies, which were sitting somewhere, and some people would have pocketed it" (Regional Manager, July 22, 2011).

"Many scheme managers have built houses, many healthcare providers have built houses, and many pharmacists have built houses in no time because there are too many loopholes in the system that people can tap in. I can treat you for one disease and bill you for something else that I deem fit; there is no way you would know...If I am in the same house with you, you are sick, and you do not have a card, I would take your sickness... and go and describe it as much as possible, bring the medicine to you" (Consultant, November 20, 2011).

The clinical audit system has since helped to reduce the level of fraud and corruption in the scheme.

Concerns were also expressed about client or provider 'shopping,' a process whereby a subscriber moves from one provider to the other with the same sickness within a short period of time or within the same day with the aim of securing medicine which they then can sell. Even though mechanisms have been put in place to check for abuse, the fact that health providers are not networked makes it difficult for service providers to tell whether a patient has been to another facility or what medicines have been prescribed.

"A client can move from one provider to another within a day. He or she collects drugs here in SDA hospital, move to Central Hospital, collects drugs, go to Teaching Hospital collects drugs, put the drugs together and sell it to a quack doctor or a quack chemical store and make money. That kind of thing, client shopping ... to make money. That is our difficulty we are facing and is threatening the scheme" (A Regional Manager, July 22, 2011).

During the interviews, a donor partner pointed to the World Bank allocation of US $\$ 15$ million to put in place a technology mechanism to help address the issue as an indication of the seriousness of the problem. This was under a project designed 
in 2007 to be called the Health Insurance Project to help ensure efficient systems are put in place for implementation of the health insurance. The interviewee explained that:

"Issues of cost containment, issues of actuarial analysis, issues of coverage of the poor, issues of electronic claims are all the things that are being done under our project to support the health insurance scheme" (Donor Partner, December 1, 2011).

Explanations offered by participants centred on the loopholes within the system such as lack of effective technology that would enable service providers track clients through the health delivery chain. However, it was not confirmed if the US\$15 million was disbursed to the NHIS.

\section{Discussion}

The findings of the study identify a range of economic and political implementation issues threatening the effective working of the scheme and its sustainability. However, similar economic and political challenges were identified across the four DMHISs in the two regions.

Economically, as in common with many developing countries, in Ghana a relatively low proportion of the working population is in formal employment. Over $80 \%$ of the population are employed $^{33}$ in the informal sector. Reliable income assessment for premium or contribution purposes is challenging and hence subscribers do not pay according to ability as insurance principles demand. Meanwhile, the design of the scheme is such that there is an inherent vertical equity in contributions, where the rich and healthier people are required by law to pay more to support the less healthy, poor and vulnerable. ${ }^{15}$ However, application of this legal requirement to the large informal sector employees and the self-employed has been administratively challenging due to fluctuations in their incomes and lack of data. ${ }^{15}$ The consequence of this is low revenue as premium contributions are less than $5 \%$ of the NHIS income. This finding is consistent with Mishra et al study in Nepal where they posited that SHI revenue collection in large informal sectors is a major economic challenge to successful implementation. ${ }^{4}$ It is also an indication that the scheme survives largely on the health insurance levy of $2.5 \%$ tax on goods and services, accounting for about $70 \%$ of revenues ${ }^{28}$; making sustainability a challenge as it has not been increased in spite of the growth in membership. The low level of contributions and what was seen as poorly targeted exemptions were argued to be a threat to sustainability, which has been noted as a major challenge in large informal economies. This is consistent with the challenge of revenue collection noted by International Bank for Reconstruction and Development (IBRD)/World Bank in low-income countries. ${ }^{8}$ There are also financial pressures resulting from problems with the claims management and payment system and, especially, provider reimbursement delay, confirming findings of other studies. ${ }^{1,46,47}$

Health insurances are established to provide financial protection against catastrophic healthcare expenditure for households. Measures such as exemptions are incorporated to ensure the poor, children; the aged and other vulnerable groups are covered. In this regard, the Ghana scheme has done extremely well. However, evidence from the study shows that the exemption policy to a large extent partly contributes to the economic challenges facing the scheme. While scaling up coverage is important to protect majority of the population and to increase universal access, the policy has not been target-specific enough resulting in dire economic implication in terms of mobilising adequate financial resources. Commenting on the economic implications of the exemption policy, for instance, the CEO of the NHIA admitted that something is fundamentally wrong if premiums constitute less than $4 \%$ of the NHIS revenue. Contributions "have often come in below 5\%, it is $3.4 \%$ and if premium collection accounts for less than 4\%, you could tell that there is something fundamentally wrong." ${ }^{\text {"[2] }}$ Meanwhile, it is acknowledged in this study that a country-wide common targeting mechanism (CTM) for targeting and enrolling the poor ${ }^{15}$ is in process as way of reforming the exemption policy to make it more target-specific.

It was also discovered that as in many LMICs (and indeed developed healthcare systems), there is a culture of curative and hospital-centric care in the Ghana health service. In other low- and middle-income and some developed countries, perception of poor quality service at the lower level means that secondary and tertiary hospitals often accommodate patients that ought to be treated at hospitals at levels below or above them. Thus, the challenge as noted in the results section is the poor gatekeeping system due to weakened checks and balances $^{12,29}$ and the lack of incentives or motivation on the part of service providers to enforce it resulting in what was described as client shopping. Yet curative or hospital-centric care is expensive all over the world. For instance, in Brazil, it is estimated that hospitals absorb nearly $70 \%$ of public spending on health ${ }^{48}$ and the case of Ghana might not be different. Such huge expenditure definitely impacts on the financial sustainability of the health insurance scheme, especially, services rendered at secondary and tertiary levels. Policy-makers needed to anticipate increases in utilisation and incorporated preventive measures.

In relation to the above, the study also establishes the potential of selective enrolment of high risk or less healthy individuals into the scheme, which raises average costs on the NHIF since coverage remains less than universal. Although, since June $2013^{[3]}$ a pilot of biometric registration and instant ID card issue has been in practice, an unintended outcome, however, could be that the immediate issuing of cards may facilitate self-selection of the sick into the scheme, which may in turn increase financial pressure on the programme. The consequence is increased cost resulting from increasing utilisation paid for from the mostly tax-based financing sources, which seems to grow more slowly than enrolment and consequent use. ${ }^{13}$

Problems of fraud and corruption were argued to be particularly evident in the early stages of implementation of the Scheme (in the form of what participants described as client/provider shopping) with some indication from interviewees that there has been improvement in more recent years. The study acknowledges that fraud is a challenge for insurance systems the world over. Steps taken to prevent and identify these problems, include the mentioned US\$15 million World Bank grant to boost the technology capacity of the 
authority and providers and to facilitate electronic processing of claims and to help track visits by subscribers. The problem, meanwhile, is whether the fund was disbursed. Findings of the study still suggest a continuing lack of effective technological monitoring and control mechanisms. The cumulative effect of this is the exploitation of the weaknesses of the inefficient technological monitoring and control mechanisms by some service providers and scheme managers to the disadvantage of the scheme in terms of financial sustainability and poor gatekeeping system. This results in abuse of the system financially but also increases the tendency of moral hazards. Politically, there is government's reluctance to increase the health insurance levy since implementation due to fear of political backlash. Meanwhile, as noted by Carrin et $\mathrm{al}^{49}$ and Doetinchem et $\mathrm{al},{ }^{50}$ government stewardship is key to the success of such systems and the welfare of the population. ${ }^{49,50}$ Political nepotism has also come for mention, especially, with regards to recruitment procedures where the scheme has become vehicle for political rewards of party loyalists. This has undermined the capacity and capability of scheme staff in terms of skills, understanding of policy standards and goals and the ability to develop micro-level programmes in response to macro-level plans. Prior studies of implementation challenges of the NHIS in Ghana have also identified politicisation as a major challenge resulting in people not joining the scheme because they either associate the scheme with a political party or a particular politician in office. ${ }^{28}$ Added to this is the rapidity with which policy decisions are made as implementation progresses. Indeed, as suggested by an earlier study, the lack of policy clarity and frequent changes in the policy made the scheme confusing to potential members, ${ }^{28}$ thereby undermining enrolment and renewals and hence the finances of the scheme.

\section{Policy Implications}

Within the context of this study and other research on the NHIS in Ghana and available data, it is suggested that addressing political and economic challenges of the NHIS in Ghana requires a policy review of the exemption policy to make it more target-specific. This has the potential of ensuring those who really cannot afford get services. Contributions from the informal sector employees also need to be marginally adjusted so as to ensure the government continue to subsidize the cost of healthcare in Ghana. It is also the view of this study that the health insurance levy should be reviewed upwards by at least $1.5 \%-2.0 \%$ as a way of matching revenue with increased utilisation and membership. Some percentage of taxes could also be levied on alcohol and Tobacco as is done in countries such as Belgium and the United Kingdom and the proceeds channelled into the NHIF as additional sources of funding. To deal with the political challenges, the study takes the view that recruitment into the scheme should reflect competence and experience rather than other considerations such as political affiliation as a way of dealing with the capacity challenges confronting the scheme.

\section{Limitations of the Study}

We acknowledge that the findings of the study cannot be generalised beyond the four case study areas as the study covered two mutual schemes each in the Greater Accra and Northern regions of southern and northern Ghana, respectively. Finally, the study covered the implementation process from 2004-2013 but it is acknowledged that implementation is a complex process that can be affected by both endogenous and exogenous factors beyond the control of implementing officials.

\section{Conclusion}

The study explored the implementation of the NHIS in selected districts in southern and northern Ghana using two mutual schemes each in Greater Accra and Northern regions, respectively, to demonstrate the implementation challenges. The study establishes that political and economic factors have influenced the implementation process and the degree to which the policy has been implemented as intended. This is not surprising as the study establishes that there is a synergy between implementation and politics. Implementation cannot be devoid of politics as it requires the necessary political authority to succeed. Thus, to achieve UHC under the NHIS requires political stewardship. Political leadership has the responsibility to build trust and confidence in the system by providing the necessary resources and backing with minimal interference in the operations.

\section{Acknowledgements}

I am grateful to the Vice Chancellors Research Studentships Committee and the Faculty of Social Sciences, University of Ulster, Coleraine, UK for sponsoring the studies, the Social Sciences Research Graduate School for funding the research fieldwork project. I also wish to acknowledge Dr. Gordon Marnoch and Dr. Ann Marie Gray, both of the School of Criminology, Politics and Social Policy, University of Ulster for all the support during my $\mathrm{PhD}$ studies.

\begin{abstract}
Ethical issues
Ethical approval was gained from the University of Ulster Research Ethics Committee, Coleraine, UK and from the Ghana health service. Permission was also obtained from the NHIA to visit the selected DMHISs. Participants were provided with information sheet about the study. Signed written informed consent was obtained from individual participants, as was permission to audio record the interview prior to the in-depth interviews. Reporting of data and findings has been strictly confidential and anonymous. Participants were also informed through the information sheet that participation was voluntary and they could withdraw at any point in the interview. Two participants availed themselves of this by withdrawing in the course of the interview so as to attend to other equally important matters, while one participant declined to be audio-recorded. All identifying features of participants were kept separate from the main data in order not to breach our guarantees with respect to anonymity.
\end{abstract}

Competing interests

Author declares that he has no competing interests.

Author's contribution

$\mathrm{AF}$ is the single author of the paper.

\section{Endnotes}

[1] Gbungbaliga is a poor farming rural community in the NSD of the Northern Region of Ghana.

[2] Interview in October 2012 on http://www.myjoyonline.com.

[3] Press Release: NHIA Begins Biometric Registration and instant NHIS ID Issuance at: http://www.nhis.gov.gh/_Uploads/dbsAttachedFiles/30(1).pdf. Last accessed on August 1, 2013. 


\section{References}

1. Sakyi EK, Atinga RA, Adzei FA. Managerial problems of hospitals under Ghana's National Health Insurance Scheme. Clinical Governance: An International Journal. 2012;17(3):178190. doi:10.1108/14777271211251291

2. Baltussen R, Bruce E, Rhodes G, Narh-Bana SA, Agyepong I. Managing mutual health organisations in Ghana. Trop Med Int Health. 2006;II(5):654-659. doi:10.1111/j.13653156.2006.01621.x

3. World Health Organization (WHO). Social health insurance: sustainable health financing, universal coverage and social health insurance; 2005 16-25 May. Geneva: WHO; 2005.

4. Mishra SR, Khanal P, Karki DK, Kallestrup P, Enemark U. National Health Insurance Policy in Nepal: challenges for implementation. Glob HealthAction. 2015;8:28763. doi:10.3402/gha.v8.28763

5. Ekman B. Community-based health insurance in low-income countries: a systematic review of the evidence. Health Policy plan. 2004;19(5):249-270. doi:10.1093/heapol/czh031

6. De Allegri M, Sauerborn R, Kouyaté B, Flessa S. Community health insurance in sub-Saharan Africa: what operational difficulties hamper its successful development? Trop Med Int Health. 2009;14(5):586-596. doi:10.1111/j.13653156.2009.02262.x

7. Agyemang KK, Adu-Gyamfi AB, Afrakoma M. Prospects and challenges of implementing a sustainable national health insurance scheme: the case of the Cape Coast metropolis, Ghana. Developing Country Studies. 2013;3(12):140-148.

8. Preker AS, Scheffler RM, Bassett MC. Private voluntary health insurance in development: friend or foe? World Bank Publications; 2007.

9. Preker A. Health care financing for rural and low-income populations. The Role of Communities in Resource Mobilization and Risk Sharing; 2003.

10. Carrin G. Community based health insurance schemes in developing countries: facts, problems and perspectives. Geneva: WHO; 2003.

11. Carrin G, James C. Reaching universal coverage via social health insurance: key design features in the transition period. Geneva: WHO; 2004.

12. Agyepong IA, Adjei S. Public social policy development and implementation: a case study of the Ghana National Health Insurance scheme. Health Policy Plan. 2008;23(2):150-160. doi:10.1093/heapol/czn002

13. Witter S, Garshong B. Something old or something new? Social health insurance in Ghana. BMC Int Health Hum Rights. 2009;9(1):20. doi:10.1186/1472-698x-9-20

14. Seddoh A, Adjei S, Nazzar A. Ghana's National Health Insurance Scheme: Views on Progress, Observations, and Commentary. Accra, Ghana: Centre for Health and Social Services; 2011.

15. NHIA. National Health Insurance Authority, 2013 Annual Report. Accra: NHIA; 2013.

16. Durairaj V, D'Almeida S, Kirigia J. Health systems financing, the path to universal coverage. Ghana's Approach to Social Health Protection: Background Paper, 2. Geneva: World Health Organization; 2010.

17. Alhassan RK, Duku SO, Janssens W, et al. Comparison of perceived and technical healthcare quality in primary health facilities: implications for a sustainable national health insurance scheme in Ghana. PloS one. 2015;10(10):e0140109. doi:10.1371/journal. pone.0140109

18. Mohammed A, Seidu M. Assessing the quality of primary health care provided by accredited service providers in the upper east region, Ghana. International Journal of Economics, Commerce and Management. 2014;II(10):1-32.

19. Nketiah-Amponsah E. Demand for health insurance among women in Ghana: cross-sectional evidence. http://hdl.handle. net/123456789/2224. Published 2009.

20. Mensah J, Oppong JR, Schmidt CM. Ghana's National Health Insurance Scheme in the context of the health MDGs: an empirical evaluation using propensity score matching. Health Econ. 2010;19(S1):95-106. doi:10.1002/hec.1633

21. Nguyen $H T$, Rajkotia $Y$, Wang $H$. The financial protection effect of Ghana National Health Insurance Scheme: evidence from a study in two rural districts. Int J Equity Health. 2011;10(4):1-12. doi:10.1186/1475-9276-10-4

22. Ayimbillah Atinga R. Healthcare quality under the National Health Insurance Scheme in Ghana: perspectives from premium holders. International Journal of Quality \& Reliability Management. 2012;29(2):144-161. doi:10.1108/02656711211199883

23. Odeyemi IA, Nixon J. Assessing equity in health care through the national health insurance schemes of Nigeria and Ghana: a review-based comparative analysis. Int $\mathrm{J}$ Equity Health. 2013;12:9. doi:10.1186/1475-9276-12-9

24. Blanchet N, Fink G, Osei-Akoto I. The effect of Ghana's National Health Insurance Scheme on health care utilisation. Ghana Med J. 2012;46(2):76-84.

25. Yevutsey S, Aikins M. Financial viability of district mutual health insurance schemes of lawra and sissala East districts, Upper West Region, Ghana. Ghana Med J. 2010;44(4):130-137.

26. Owusu-Sekyere E, Bagah DA. Towards a sustainable health care financing in ghana: is the national health insurance the solution? Public Health Res. 2014;4(5):185-194.

27. Mwinkume G, Obuadey G. Assessing the sustainability of the operations of the national health insurance scheme: a case of Oguaa Mansin Health Insurance Scheme, Cape Coast, Ghana. International Journal of Innovative Research and Development. 2014;3(5):649-657.

28. Gajate-Garrido G, Owusua R. The national health insurance scheme in Ghana: Implementation challenges and proposed solutions. http://papers.ssrn.com/sol3/papers.cfm?abstract_ id=2373242. Published 2013.

29. Imurana BA, Haruna RK, Nana Kofi AB. The politics of public policy and problems of implementation in Africa: an appraisal of Ghana's National Health Insurance Scheme in Ga East District. International Journal of Humanities and Social Science. 2014;4(4 Special Issue):196-207.

30. Abiiro GA, Mclntyre D. Universal financial protection through National Health Insurance: a stakeholder analysis of the proposed one-time premium payment policy in Ghana. Health Policy Plan. 2013;28(3):263-278. doi:10.1093/heapol/czs059

31. Abiiro GA, Mclntyre D. Achieving universal health care coverage: Current debates in Ghana on covering those outside the formal sector. BMC Int Health Hum Rights. 2012;12:25. doi:10.1186/1472-698X-12-25

32. Patton MQ. Qualitative Research and Evaluation Methods. Thousand Oaks, California: Sage Publications; 2002.

33. GSS. 2010 Population and Housing Census. Summary Report of Final Results. Accra: Ghana Statistical service; 2012.

34. Annim SK, Mariwah S, Sebu J. Spatial inequality and household poverty in Ghana. Economic Systems. 2012;36(4):487-505. doi:10.1016/j.ecosys.2012.05.002

35. Asenso-Okyere WK. Health Financing: Financing health care in Ghana. Paper presented at: World Health Forum; 1995.

36. Owusu g. An assessment of Regional and Gender equity in healthcare coverage under different healthcare policies in Ghana. Ghana Journal of Geography. 2014;6:42-62.

37. Adu-Gyamfi AB, Abane AM. Victims of location: Access to health care in the Lake Bosomtwe Basin of Ghana. European International Journal of Science and Technology. 2013;2(5):1-5.

38. Commission NDP. Medium-term national development policy 
framework: Ghana shared growth and development agenda (GSGDA) II, 2014-2017. https://s3.amazonaws.com/ndpc-static/ pubication/GSGDA+II+2014-2017. pdf. Published 2014.

39. Asenso-Okyere WK. Financing health care in Ghana. World Health Forum. 1995;16:86-91.

40. Aryeetey GC, Jehu-Appiah C, Kotoh AM, et al. Community concepts of poverty: an application to premium exemptions in Ghana's National Health Insurance Scheme. Global Health. 2013;9:12. doi:10.1186/1744-8603-9-12

41. McCracken G. The Long Interview. Vol 13. Sage; 1988.

42. Denscombe M. The Good Research Guide: For Small-Scale Social Research Projects. UK: McGraw-Hill Education; 2014.

43. Thomas JW, Grindle MS. After the decision: Implementing policy reforms in developing countries. World Dev. 1990;18(8):11631181. doi:10.1016/0305-750x(90)90096-g

44. Sutton R. The Policy Process: An Overview. London: Overseas Development Institute; 1999.

45. Clay EJ, Schaffer B. Room for manoeuvre: an exploration of public policy planning in agricultural and rural development. Rutherford: Fairleigh Dickinson University Press; 1984.

46. Witter S, Garshong B, Riddle V. An exploratory study of the policy process and early implementation of the free NHIS coverage for pregnant women in Ghana. Int J Equity Health. 2013;12:16.

47. Arpoh-Baah B. Assessing financial sustainability of National Health Insurance Scheme (NHIS) in Ghana Case Study: Mpohor Wassa East Mutual Health Insurance Scheme; 2011.

48. La Forgia GM, Couttolenc B. Hospital performance in Brazil: the search for excellence. World Bank Publications; 2008.

49. Carrin G, Doetinchem O, Kirigia J, Mathauer I, Musango L. Social health insurance: how feasible is its expansion in the African Region. Development Issues. 2008;10(2):7-9.

50. Doetinchem O, Schramm B, Schmidt JO. The benefits and challenges of social health insurance for developing and transitional countries. Financing health care-A dialogue between South Eastern Europe and Germany. Series International Public Health; 2006. 